\title{
Safe sailing: GSM and GPS controlled autonomous boat with overweight detection and obstacle avoidance
}

\author{
Shadman Sakib Arnob, Adiba Sumaiya Khan, Rashed Shelim, Mahmood Chowdhury \\ Department of Electrical and Computer Engineering, North South University, Bangladesh
}

\begin{tabular}{l}
\hline \hline Article Info \\
\hline Article history: \\
Received Oct 23, 2018 \\
Revised Dec 24, 2018 \\
Accepted Jan 13, 2019 \\
\hline
\end{tabular}

\section{Keywords:}

Aquatic vehicle

Autonomous

Barcode scanner

Digital compass

GPRS

GSM

PID

Ultrasonic radar

\begin{abstract}
This paper aims to save thousands of lives by proposing a novel technique of ensuring the complete safety of medium-sized aquatic vehicles using innovative ideas as well as augmented adaptations of myriad existing technologies. The proposed system incorporates a warning and danger level detection circuit using transistors for switching purposes when the vehicles are overloaded, and a Global System for Mobile (GSM) based module so that the control room can receive alerts and control the engines of such vehicles centrally. A system for detecting and avoiding obstacles is made using ultrasonic radar with ultrasonic transducer JSN SR04t mounted on top of SG90 servo arm which rotates to detect any obstacles. When an obstacle is detected, two other ultrasonic sensors SR-04 gets activated which are placed on two sides of the aquatic vehicle and the ultrasonic transducer becomes fixed on the exact centre. All the three sensors work together to find a free path for the boat to travel. If there is no free path, the boat will stop and wait for the paths to get cleared. The location of the vehicle is tracked by the Global Positioning System (GPS) and a Proportional-Integral-Derivative (PID) controller has been included along with a system which uses values from the GPS module to come back to its original path if it deviates from the original path when avoiding obstacles. A barcode system has been added where it keeps a count on the passengers. The tickets for the vehicle will have barcodes on them which will let the passengers in only if the barcode matches. This is used mainly to keep track of how many people are boarding the vehicle and to prevent those without tickets from boarding.
\end{abstract}

Copyright $(0) 2019$ Institute of Advanced Engineering and Science. All rights reserved.

\section{Corresponding Author:}

Rashed Shelim,

Department of Electrical and Computer Engineering,

North South University,

Plot-15, Block-B, Bashundhara R/A, Dhaka- 1229, Bangladesh.

Email: rashed.shelim@northsouth.edu

\section{INTRODUCTION}

In a span of just 14 years from 2000 to 2014, more than 16,000 lives had been lost to ferry accidents around the world [1]. While ferries and accidents associated with them are a pressing concern in many different developing countries, the highest number of accidents usually occurs in the same country where this paper is based: Bangladesh.

Bangladesh's economy is dependent largely on its capital city of Dhaka, and as such, most workers leave their hometowns to come work in this city. Thus, during national holidays or during religious festivals, ferries and launches, brimming to the top with passengers eager to go home, set off from Dhaka towards various other cities throughout the country. The captains of these vessels usually have little regard for much else besides profit and dangerously overburden their vehicles, which often leads to sinking and loss of hundreds of lives at a time. Other problems such as collisions with smaller vessels in the fog also occur. 
Although almost all such incidents get ample media coverage and the nation mourns every time one occurs, it is quickly forgotten and no practical steps are taken to surmount the underlying causes. This paper thus attempts to devise innovative and comprehensive solutions to tackle these problems head-on.

After conducting some research, it was found that no such work had been done before for these problems. There were small individual work done on different aspects of the project like GPS tracking, water level sensing, etc but all of these were not made into a complete system. Individual papers were found related to PID control of underwater remotely controlled vehicles (ROVs) [2-3] but this control was not tried or tested on ships for obstacle avoidance.

The main priority of this project was to make the ship safe by detecting whether the ship is overweight and using a GSM Module to give the control of the ship to the control room situated in the shipyard so that the ship cannot leave the shipyard if it was deemed unsafe for the passengers. Also, the ship was planned to be made autonomous to reduce human errors and make the journey safer. The design of the ship was made in such a way that after detecting the obstacle, the ship could move on its own to avoid it and come back to its original path. The obstacle avoidance was done using a network of ultrasonic sensors which gave the ship 1800 view of the front and the ship would know what was in front of it and choose a suitable path to avoid the obstacle. After avoiding the obstacle, the ship would return to its original path using a PID control algorithm [4].

\section{RESEARCH METHOD}

The system that is implemented is simple, cost efficient and can be implemented on small and medium sized marine vehicles. For the purpose of implementation, we used a remote controlled boat to implement the circuit and simulate the results. The features can be divided into three main parts as shown below in the flowchart: overweight detection, obstacle avoidance and GPS tracking.

Overweight detector is a simple circuit which has four nodes where each pair provides two different levels; warning level and danger level. Warning level alerts the boat of being overweight and turns on the alarming buzzer. Danger level will turn alert the control room who can then turn off the engine remotely. Both the levels are controlled by a GSM module (A7 Ai-Thinker), which will send warning texts to the control room and can stop the engine during danger level. The only way to turn the engine on after the boat's engine is turned off by the control room is to go out of the danger level by unloading.

The ultrasonic radar [5] is built using an ultrasonic transducer mounted on top of a servo motor which rotates over an angle of 150o. The sensors used are JSN SR04T ultrasonic transducer and two HCSR04 [6] ultrasound sensors on each side of the boat to provide a wide view angle of the front. Aiming to detect the obstacles the front side transducer sensor was pinned on top of the servo at the front edge of the boat. The servo will rotate $150^{\circ}$ with the transducer to detect any obstacle. The moment it finds any obstacle, the servo will jump back in its original position and the other two sensors will be activated. Then the three ultrasonic sensors [7] will work together to determine which path is free and if the boat is deviated from its path, PID control will be used to make the boat autonomous so that it can use its current and previous locations values taken from the GPS module to determine how much it has been deviated and will use these information to come back to its original path towards the destination.

GPS tracking is done to know the location of the boat at any time and it can be used for various functions. The control room can also request for the location of the boat at any time. The boat will send the control room its location. The location is given by the GPS module and the communication between the boat and the control room is achieved using the GSM module. The boat deviates from its original track in order to avoid obstacles. The value of the deviated angle is taken from the GY-271 HMC-5883L 3-axis magnetic electric compass module [8]. PID control is used which takes the initial angle before the boat was deviated and the final location after the obstacle is avoided. Then the PID control offsets the deviation by working on a closed loop where the deviation is considered as an error signal and the closed loop feedback continues till the error signal becomes zero. When the error signal is zero, the boat has returned to its original path towards the destination.

A barcode scanner has also been used to scan the barcodes that are printed on the tickets to check whether the ticket is for this vehicle or not. Its main purpose is to keep count of how many passengers have boarded the ferry, and prevent additional people from boarding. The barcode scanner is controlling the gate, which is made using a servo and will only open if the correct tickets are scanned. Otherwise, the gate will remain closed and not allow passengers to enter through the gate. This is an added safety measurement which could also be useful to reduce the risk of becoming overweight. The system flowchart has been summarized in Figure 1.

Indonesian J Elec Eng \& Comp Sci, Vol. 14, No. 2, May 2019 : 715 - 724 


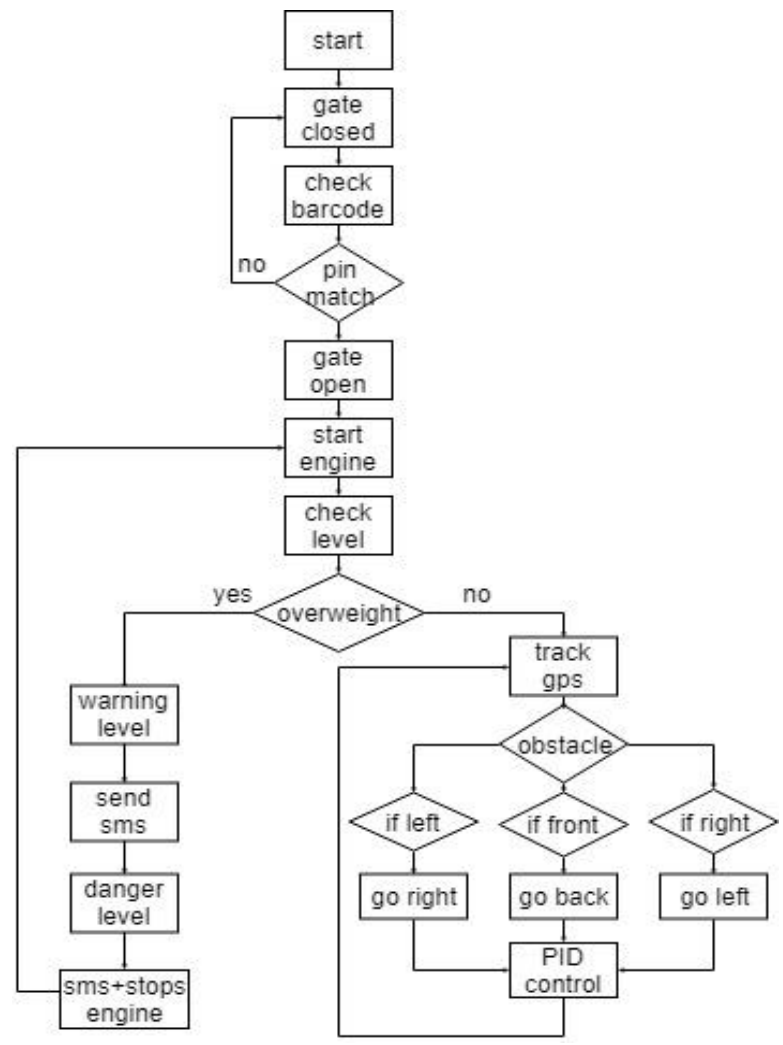

Figure 1. System flow chart

\section{RESULTS AND ANALYSIS}

The working pronciple, expected results and actual results obtained are discussed below. The main goal was not only to design a model with the intended features but also to make the simple efficient, cost effective and as simple as possible so that it can easily be implemented. The entire circuit of the project was implemented using two Arduinos- one Arduino Uno and one Arduino Mega. The Arduino Mega was the master Arduino and the Arduino Uno was the slave Arduino. The Slave Arduino had the GSM module and GPS module connected to it and was controlled by some pre-specified signaling pins those inputs came from the master Arduino. All the rest of the components and features of the project were implemented on the master Arduino including the autonomous control of the boat. The Arduino Uno had to be kept as the GPS module used was only compatible with Arduino Uno. So the GPS and GSM modules were kept together and were controlled by signals coming from the Arduino Mega.

\subsection{Sub Overweight Detection}

Boats and ferries often have a line drawn near the base which portrays how safe the boat is, i.e. if the line goes below water, the boat is said to be overweight. Based on this, an enhanced system was conceived that is as ingenious as it is simple. The designed system works in two levels: a warning level, and a danger level above it, as depicted in Figure 2.

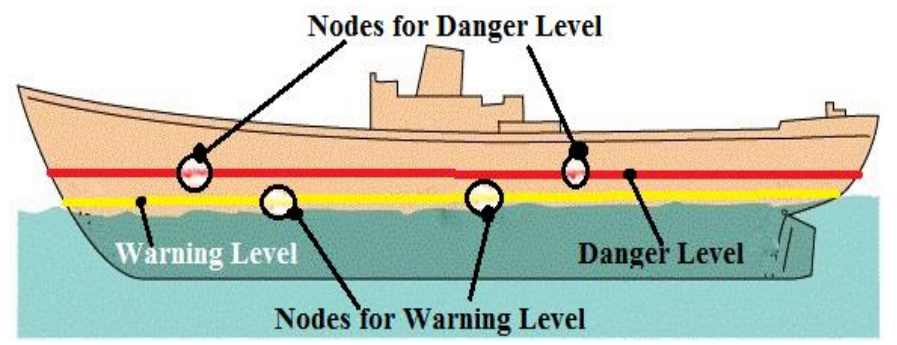

Figure 2. Overweight detection node placement 
Four NPN transistors (BC 548) are used on each level along with two nodes on either side and are placed close to a $5 \mathrm{~V}$ positive signal, but are not connected. When water reaches one of the levels, the conductive property of impure water ensures that the circuit is complete, and the current from the $5 \mathrm{~V}$ supply reaches the nodes which then send a positive signal to the base of the transistor, effectively turning it on. The transistors act like a switch and send positive signals from the collector to the emitter and this signal goes to an Arduino.

When the warning level line is submerged and all four transistors of the warning level are thus switched on, the warning is activated and an alarm goes off inside the vehicle. The alarm ensures that the people on board are also aware that the vessel is overweight. Also, a Short Message Service (SMS) is sent to the control room to alert them of it via an A7 Ai-Thinker module. This module uses a Subscriber Identity Module (SIM) card for sending and receiving text messages.

The danger level also consists of four nodes which are attached to the bases of transistors, and works in the same way as the warning level. However, when both warning level and danger level are submerged, the Arduino does not send any warnings to the ferry but it sends a danger level SMS to the control room. Then if the control room decides to ensure that the ship does not leave the dockyard, it can sends a return SMS with a specific code. The A7 Ai-Thinker would then pass this code on to the Arduino, which would read that SMS, and if the code is found, the engine is switched off and it cannot be turned on normally, but would turn on only if load from the ship is decreased and the danger level comes out of the water.

Wires or nodes were included on both sides of the boat to ensure that a false alarm is not triggered when stray waves complete the circuit on one side without the boat actually being submerged in the water. Furthermore, a timer delay has also been used so that when waves crash into the side of the boat and momentarily complete the circuit, we do not get any false positives, as it waits for the signal pulse from both sides to remain constant for a minute, prior to taking any action.

Practically, the signalling voltage retrieved from each node gave a voltage value of $3 \mathrm{~V}$. The signalling voltage was taken across the emitter resistor. The $2 \mathrm{~V}$ from the supply was dropped due to the resistance of the wires and the breadboard and the knee voltage of the transistor.

\subsection{Obstacle Detection}

Atnight, and particularly in foggy weather, ferries often collide with smaller boats and other objects leading to accidents and often death. Thus in order to automatically detect obstacles and avoid them, we have used a JSN SR04T ultrasonic transducer mounted on top a servo arm, and two HC-SR04 ultrasonic sensors on the sides to provide a cost-efficient, power saving and wide angle range for obstacle detection. The positioning of the sensors has been shown in Figure 3.

The two ultrasonic sensors are placed at North-East and North-West with respect to the boat. They are powered by the digital pins of microcontroller and are thus usually in power saving mode, i.e. there is no power consumption when it is not active. The servo arm rotates through an angle of $180 \mathrm{o}$ and the ultrasonic transducer continuously sends out pulses and waits for the echo in order to calculate the distance.

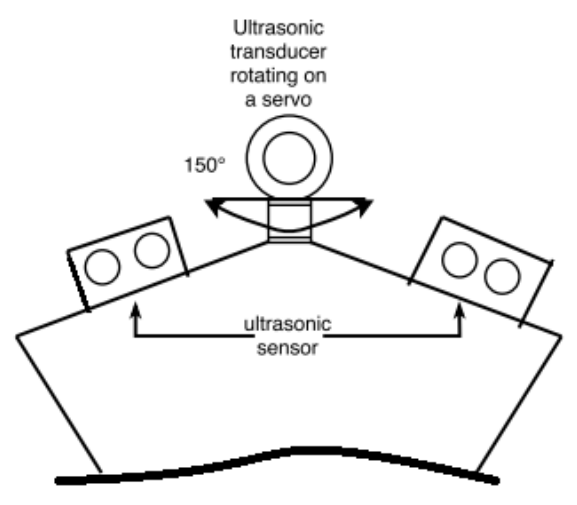

Figure 3. Front view of sensor positions

When an obstacle is detected, the servo comes back to the middle and the other two ultrasonic sensors get activated. The GPS location is also saved. Using the data from the sensors, the best path with the least obstacles is then selected and the vehicle changes direction accordingly. The system has been designed 
such that the highest priority of direction is always forward, followed by left and finally right. That is, if an obstacle appears in front of the vehicle, it will check the left side (Sensor 2), and if the left side is free, it will veer left. But if there are also obstacles to the left, then it will check if the right side is free (Sensor 3), and then veer right. If all sides are blocked, the boat will stop. In order to stop boats, sometimes back thrust is needed and so the back thrust is provided until the boat stops.

The system also saves the time for which it went left or right so that it can counter this deviation by going in the opposite direction later. That is, if for example, the vehicle veered left for ten seconds, it will then go right for ten seconds to counter the movement to the left and keep the overall direction the same. The PID controlling part is described in the next segment.

The practical implementation and arrangement of sensors are as shown in Figure 4. Through continuous experimentation, it was found that, the maximum value of the range of the ultrasonic sensors when used in the practical setup was $30 \mathrm{~cm}$ as it was placed on the miniature ship at an angle of 250 tilted towards the surface of the water as shown in Figure 5. This maximum distance of detection varied when the ship submerged in water. The maximum detection of the ultrasonic sensors were decreased a lot compared to the actual range of the sensors which was found at $200 \mathrm{~cm}$ because the sensors were tilted and after a certain distance it started to detect the surface of the water. The ultrasonic sensors were tilted so that it could also detect small boats coming in its range as the size of the boats could be smaller than the size of the ship in which the ultrasonic radar is placed.

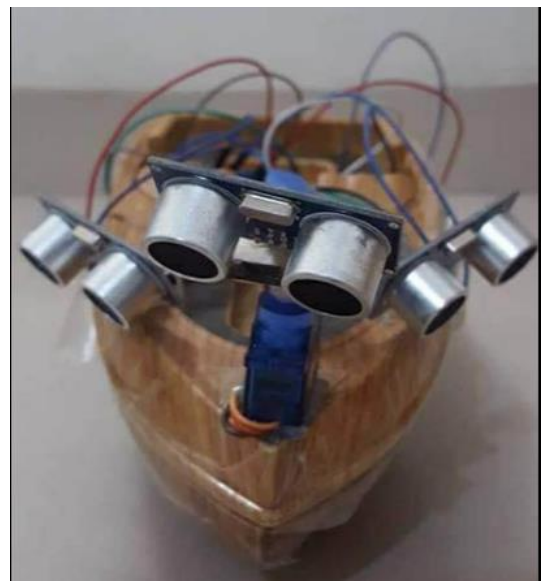

Figure 4. Front view of ultrasonic radar

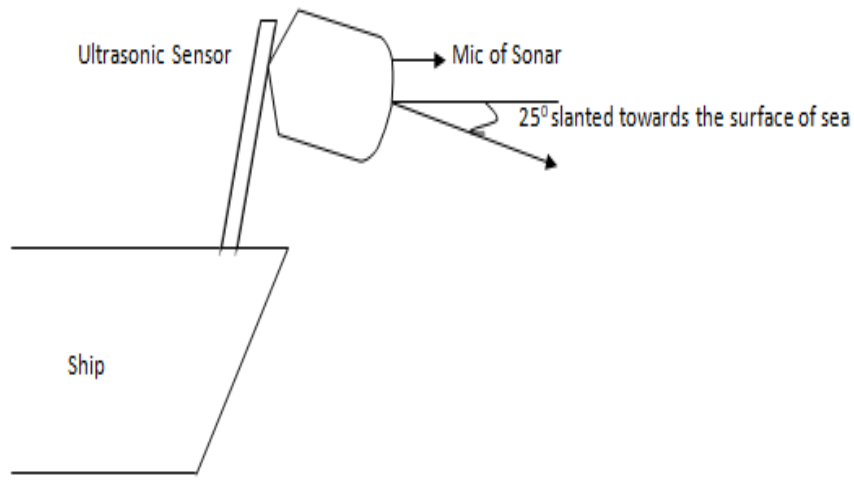

Figure 5. Side view of sensor positions

\subsection{GPS Tracking}

The boat is constantly monitored using the GPS module NEO 6M V2. It is always active and can give the location to the control room whenever the control room asks for the location. It is useful because even if someone is able to tamper with the overweight circuit, the control room can ask for the location via a particular code and the GPS will give the location of the boat. This communication is achieved using the SMS mode of the A7 GSM module.

When the boat is on its path towards the destination, it can face some obstacle for which it has to deviate from its path. Just before the deviation occurs, the microcontroller will save the current angle of the location from the electric compass and after the obstacle is avoided, it will use again the electric compass to find its new position. Then it will input these data into the PID loop equations where, by using a closed system feedback path, the boat will detect the deviation from path and will automatically come back to the original path by offsetting the change. PID is used largely due to its simple tuning method for industrial applications.

The location is being constantly detected by the GPS module. The values it is saving are nothing but coordinates found from the latitude and longitudinal axis of the Earth. By doing simple mathematical calculations, the deviated angle is found. The calculations are shown below.

Suppose the PID control for the boat is achieved by using software [9]. The constant $\mathrm{kp}$ is the proportional constant and gives output which is proportional to current error. Here the current error is the angle deviation from the track. The input is taken from the GPRS location. It compares the desired or set point with actual value or feedback process value. If the value is zero, then this controller output is zero. The working principle of PID has been shown in Figure 6. 


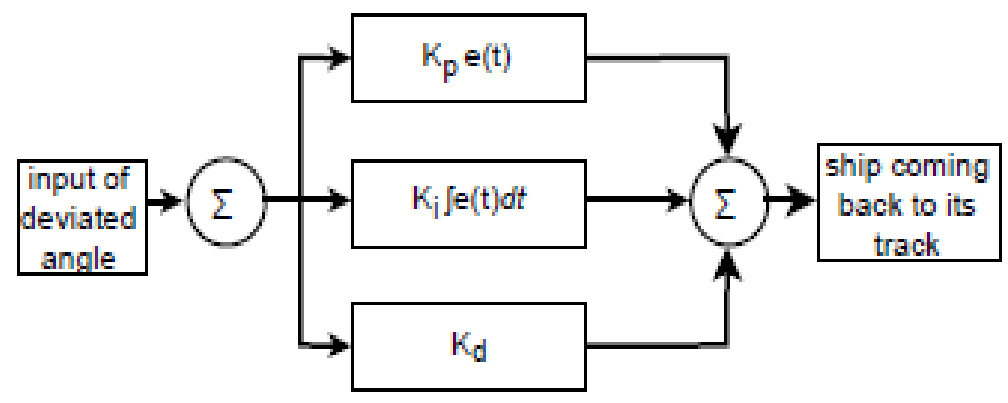

Figure 6. Block diagram of PID

Let, $a(t)=$ the angle deviated due to obstacle detection.

$k_{p} \infty a(t) \mathrm{kp}$

The constant ki is the integration constant which integrates the process variable which is the angle deviated and set point to eliminate the steady value. Integral limits the speed of response and affects the stability of the speed.

$$
k_{i}=\int a(t) d t
$$

The constant $\mathrm{kd}$ is the differentiation constant which predicts the future behaviour of the error. Its output depends on the rate of change of the error with respect to time, multiplied by the derivative constant.

$$
k_{d}=\frac{d a(t)}{d x}
$$

The error values are used to adjust the speed of the two motors so that it comes back to the original path. The overall working principle of the PID control algorithm is as shown in Figure 4. A closed loop feedback is used to change the speed of motor so that the boat comes back to the original path. The change in speed of the motor is determined by the error signal. Whether the signal is positive or negative determines which motor's speed will be altered.

As input for the PID control, we used the angle found using the latitude and longitude values given by the GPS module. When an obstacle comes across the path, the ship will change its direction creating a deviated angle. The values that will be given as input are the angle it is supposed to be headed (P0) and the deviated angle $(\mathrm{Pn})$.

The error signal, $\mathrm{e}(\mathrm{t})$ is given by:

$$
\mathrm{e}(\mathrm{t})=\mathrm{Pn}-\mathrm{P} 0
$$

Then the speed of the motor is changed by the derived closed loop PID equation which is applicable to our system:

$$
\text { New Speed }=\frac{e(t)}{k_{P}}+\frac{e(t)-\dot{e}(t)}{K_{d}}+\frac{e_{S}(t)}{K_{i}}
$$

Where,

$\dot{e}(t)=$ previous error

$e_{s}(t)=$ sum of errors

This new speed is then fed into the motors driving the ship and gets added with the previous motor speed values so that the speed of the motor changes appropriately and the ship comes back to the original position. Additional precautions will be taken so that the speed of the motors do not exceed beyond a certain value and do not fall below 0 . This process gets repeated again and again till the error becomes zero, at which point the boat's motors run at the same speed and so the boat is moving forward. As the error becomes 0 , the boat has returned to its original path. The PID constant values were taken as shown in Table 1 . During experimentation, the following values of the PID constants were used: 


\begin{tabular}{cccc}
\multicolumn{4}{c}{ Table 1. PID Constant Values } \\
\hline Serial No. & $\mathrm{K}_{\mathrm{P}}$ & $\mathrm{K}_{\mathrm{i}}$ & $\mathrm{K}_{\mathrm{d}}$ \\
\hline i) & 5 & 500 & 1 \\
ii) & 15 & 700 & 5 \\
iii) & 30 & 800 & 10 \\
iv) & 40 & 1000 & 7 \\
v) & 35 & 950 & 8 \\
vi) & 37 & 940 & 9 \\
\hline
\end{tabular}

Trial and error method had to be used in order to determine the best possible values of the constants. From this method, it was determined that the values of the constants at serial no (vi) of the table was most suitable for our setup. However, due to the small difference in values of the starting position and current position given by the GPS module, the boat took a long time to come back even though it was in a closed loop feedback path.

In order to improve accuracy and get a significant change in change of angle, we used GY-271 electronic compass [10]. This is a digital compass module that can be used to find the angle with respect to the Earth's magnetic field. By using this module, we were able to find a significant error signal and so PID control worked better using this module. The following values of the PID constants were used. The PID constant values were taken as shown in Table 2 for the digital compass module.

\begin{tabular}{cccc}
\multicolumn{4}{c}{ Table 2. PID Constant Values } \\
\hline Serial No. & $\mathrm{K}_{\mathrm{P}}$ & $\mathrm{K}_{\mathrm{i}}$ & $\mathrm{K}_{\mathrm{d}}$ \\
\hline i) & 1 & 0 & 0 \\
ii) & 5 & 0 & 0 \\
iii) & 10 & 0 & 0 \\
iv) & 9 & 1 & 0 \\
v) & 9 & 5 & 0 \\
vi) & 9 & 5 & 1 \\
vii) & 9 & 1 & 5 \\
\hline
\end{tabular}

It was determined that the values of the constants at serial no. (iv) of the table was most suitable for our setup using the digital compass module. So for this setup, the best option was to use a PI controller. As the error signal found had a significant change, the ship turned back to its original path at a faster pace. The oscillation of the ship due to PI control was also very low when the values of the constants at serial no. (iv) of the table was used. The time it took to return to the original heading depended on the amount of deviation it went through. It was practically found that the ship took around 3.5 seconds to come back to its original heading for every 900 change in angle and the maximum possible angle of deviation was 1800 as the ship could turn through either direction to come back to its original heading.

\subsection{Barcode Scanning}

A barcode scanner is used to keep track of the people who have boarded the ferry, as when buying the tickets the person would have to provide personal information and also some sort of identification card (e.g- National ID card, driving license, passport, etc.). Barcodes data can be used as automatic identification and data capture. It captures data from varying the width and from the parallel lines [11]. This would help in knowing the name and information of the people boarding the vessel and also keep count of the total number of people on board. The scanner would also prevent people without tickets enter the vehicle.

The barcode scanner is used to control the gate of the vessel, which is made with a servo. If the barcode matches, the servo is sent a signal to open the gate via rotating its arm through an angle of 90o clockwise. An ultrasonic sensor is used to detect the passengers going through the gate. The distance between two ends of the gate is kept at $20 \mathrm{~cm}$ and if the ultrasonic sensor detects a value less than $20 \mathrm{~cm}$, the microcontroller interprets this data to understand that the passenger has passed and rotates the servo arm through an angle of 90o anti-clockwise to close the gate. The barcode scanner used is CD-100II from Birch.

The barcode scanner used has a low power consumption of $5 \mathrm{~V}$. A database will be available where all the generated codes for the tickets will be present and the barcode scanner would scan the barcodes and run the scanned code through the database to check whether it matches with any codes from the database. The entire barcode system has been shown in Figure 7.

The issue with the barcode was that it could not be connected directly to Arduino as Arduino could not power the Barcode scanner when an USB shield was used and it could not interpret the data found after 
scanning when a PS/2 male port. The barcode readings were taken to Arduino via a computer and those readings were then used as inputs to check whether the input code matched the database or not.

Also, an emergency button was placed at the control room of the ship so that the gates can be kept open in case of emergencies where a quick evacuation of passengers would be needed. This button would automatically send a signal to the Arduino to keep the gates open till this switch was closed. Once the switch is closed, the gates would close and start working as before and so it would need barcode authentication in order to open.

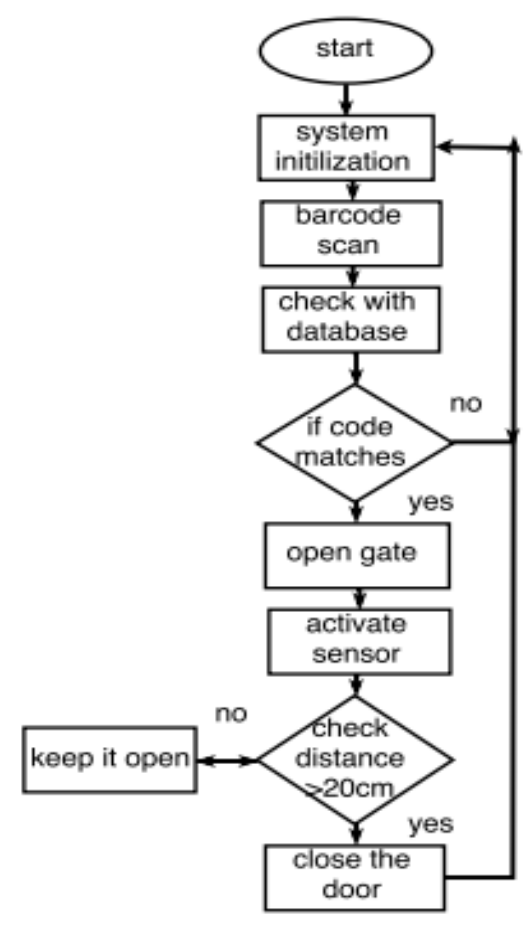

Figure 7. Barcode system flowchart

\section{CONCLUSION}

The system made was mainly done to make the ships safer for everyone so that ship accidents due to human fault could be reduced as much as possible. This initiative should have been taken long ago to ensure safety of passengers but till now nobody has taken it. The system has been designed in such a way so that ship accidents could be reduced as much as possible. Despite the comprehensiveness of the proposed system, a few features can be added to make it even more robust.

For example, there is sometimes a buildup of sediments under the water where the ferries often get stuck. This can be avoided by installing an underwater depth sensor [12] so that whenever the depth seems too low, the control room can be alerted via an SMS from the A7 module, and the location can be saved so that the authorities can raze these mounds at a convenient time. The module would also take measurements of the depth of water using an underwater distance measuring sensor and would also take the value of the location with the GPS module so that it can keep track of the depth of water at particular places. This would be stored in an offline database and after the boat reaches the shore, the database can be taken by the control room that would keep track of the depth of water at each particular point. This would be useful as a lot of boats and ships can get stuck in the middle of the sea and also accidents can occur.

Further work can be done on the barcode scanner so that it can directly connect with Raspberry Pi and not use an intermediate device like a computer to take readings. If a Raspberry Pi is used all the features could work under it and would not need master-slave Arduinos.

The design and ease of implementation of the system implemented could have a lot of impacts. Although overshadowed by the prevalence of much more significant factors, a ferry or water vessel sinking also leads to significant water pollution. The fuel of these vessels also harms the underwater fauna, and the system ensures that such a scenario is prevented. The economic impact of the proposed system is multifaceted, in that many business owners will feel that they are being cheated out of money by the 
restriction on passengers that we have imposed. However, the wise businessmen will realize that while it may seem that they are giving up profit, they are actually saving millions on the hundreds of vessels that would have otherwise sunk and eradicated any scope of further profit. The components used to set up the system are all readily available. Thecomponents can be bought in bulk at a low price, and if PCB is used, it becomes all the more efficient and easy to assemble. Thus, it can be concluded that the manufacturability is quite high. As long as the equipment is kept in specialized compartments such that water does not reach it, there is no reason for it to be harmed. In case of sensor failure or other technical problems, the problematic components can easily be replaced. We thus conclude that each system should ideally last a significant amount of time without any problems.

The cost of the overall project was taken into consideration at every step. The desired features that were to be implemented needed to be as efficient and low cost as possible. Maintaining a reasonable cost was one of the main goals as it would not be implemented on only one ship but on thousands of them if the project was given permission to be implemented on actual ships. So, it was necessary to make an overall package with all the features of the project that could be replicated on actual ships and the cost also had to be as low as possible so that the ship owners become willing to implement the system on their ship. As there were no other systems like this in the market, the project would be pioneering the implementation of this system and design. So even with so many features, it was mandatory that the system to be as cheap as possible so that it can become available to all the ship owners.

A novel technique of ensuring the safety of ferries and other marine vehicles has been presented in this paper. The proposed system consists of three separate parts. Firstly, an overweight detection system and a means to remotely control the engine have been designed that functions using an A7 Ai-Thinker Module, and utilizes the conducting property of impure water. In addition, an obstacle detection system has been included which works using three different sensors and codes to automate the process of avoiding obstructions. Furthermore, a GPS system has been incorporated which has a plethora of functions including ensuring that an overweight ferry does not leave the dock, and using PID to keep the vehicle on track even after any deviations to avoid obstacles. Lastly, a barcode security system has been implemented which only allows people with tickets to enter, and keeps track of all people on board at any given moment. The general purpose of the system goes well beyond the realms of academics and has the potential, if implemented correctly, to save thousands of lives.

\section{ACKNOWLEDGEMENTS}

Firstly, we would like to thank Md Mahbub Alam for providing the public alarm system for the vessel. We would also like to thank fellow students of North South University Abrar Ahnaf Abid, Sifat Rezwan Tamim and Debnath Banik for their valuable insight during the course of this project.

\section{REFERENCES}

[1] A. Golden, J. Drew, 2000-2014 "Ferry Fatalities: Statistics and Causation of Major Accidents", [online] Availabe at: https://www.ferrysafety.org/news.htm [Accessed: 30 March 2018]

[2] R.S. Soman et.al, "MIMO PID Compensation for Dynamic Positioning of a Ship", in 2018 Indian Control Conference, pp. 137-142

[3] S. Garg et.al, "Autonomous Ship Navigation System”, in 2013 Texas Instruments India Educators' Conference, pp. 300-305

[4] Z. Yinhai, et al., "A Novel SVPWM Modulation Scheme," in Applied Power Electronics Conference and Exposition, 2009. APEC 2009. Twenty-Fourth Annual IEEE, 2009, pp. 128-131.

[5] M.V.Paulet et.al, "Ultrasonic Radar", in 2016 EPE Conf., pp. 551-553

[6] Ultrasonic Sensor, "HC-SR04 datasheet". [online]. Available at www.satistronics.com [Accessed: 03 March 2018.

[7] S.Zhang et.al, "Design Of Solar Ship Model with Underwater Ultrasonic Ranging”, in 2016 IEEE Int. Conf. Communications,pp. 2858-2859

[8] Electronic Compass, "GY-271 Electronic Compass Datasheet". [online]. Available at www.robotpark.com [Accessed: 21 April 2018]

[9] T. Agarwal, n.d, "Working of a PID Controller" [online] Available at: https://www.elprocus.com/the-working-of-apid-controller/ [Accessed: 30 March 2018]

[10] C. Marcu et.al, "Robot Orientation Control Using Digital Compasses", in 2013, IEEE, pp. 331-336

[11] A. Abdelnour, "Transformation of Barcode Into RFID Tag, Design, and Validation", in 2018 IEEE Microwave and Wirless Components Letters, pp. 1-3

[12] S. E. Borujeni, "Ultrasonic underwater depth measurement", Proceedings of the 2002 International Symposium on Underwater Technology. IEEE, pp. 33-36 


\section{BIOGRAPHIES OF AUTHORS}

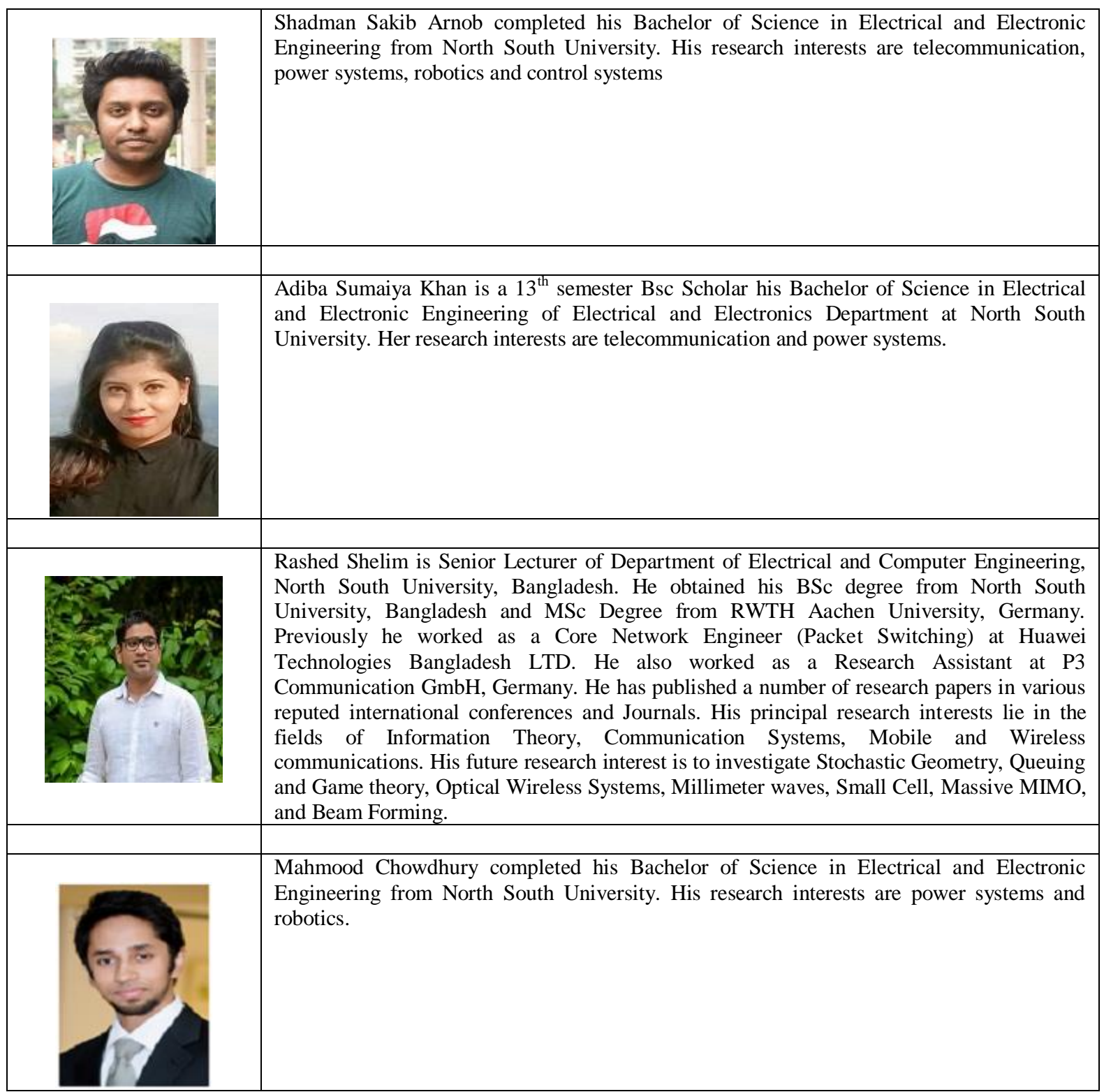

